\title{
Hydatid cyst of the liver with intrathoracic rupture
}

\author{
DS XANTHAKIS, E KATSARAS, M EFTHIMIADIS, G PAPADAKIS, \\ G VAROUCHAKIS, C ALIGIZAKIS
}

From the Thoracic Surgical Clinic, Sismanoglion Hospital, Athens, Greece

ABSTRACT In the last 10 years we have operated on 33 cases of hydatid cyst of the liver with intrathoracic rupture. Twenty-one out of 33 cases ruptured into bronchi, in seven the rupture affected the pleural cavity, and in six simultaneous rupture into the bronchus and pleural cavity occurred. Laboratory tests were not carried out in all cases. The Casoni intradermal test was carried out in 23 cases and was positive in 18. A liver scan was performed in 10 and was positive in all. Conservative operations were carried out in 22 patients. In these the hepatic cavity was evacuated and separately drained. This was followed by suturing the diaphragmatic rupture and also closing the bronchial opening if present. Lung resections were performed in 11 out of 33 cases. In eight lobectomy was carried out and in three segmental resections. Resection was necessary when suppuration and bronchiectatic changes affected the lung. Ruptured cyst into the pleural cavity requires emergency thoracotomy after the anaphylactic shock is over. Removal of the parasite, re-expansion of the lung, and drainage of the pleural and hepatic cavities is necessary. Immediate and late complications occurred in 13 patients. In two postoperative haemorrhage occurred and in two postoperative empyema developed. Recurrent haemoptysis was seen in five, persistent bile fistula in one, and dissemination of hydatid cyst in three. In the remaining 20 cases there was no complication. Operative mortality was nil.

Hydatid cysts situated in the upper liver surface have a tendency to grow upwards and if uncomplicated they may find their way through the diaphragm and become intrathoracic. Symptoms may be caused either by the size of cyst or, more commonly, if complications arise, by its rupture.

Rupture may occur into the pericardium or mediastinum, the lung and bronchial tree, the pleural cavity, or the peritoneal cavity. Before rupture into the thorax, the cyst will have already ruptured into the biliary tract leading to daughter cyst formation and pyogenic infection. There is therefore a combination of abdominal and thoracic manifestations. The symptoms from the former may vary from slight digestive disturbances to epigastric pain with jaundice and enlargement of the liver. Thoracic manifestations vary with the type of rupture. Involvement of pericardium and mediastinum is very rare and in this paper we will consider only the more common ruptures into the lung and pleura. Rupture into the chest must be considered a serious complication and there are many published reports. ${ }^{1-12}$

Address for reprint requests: Dr DS Xanthakis, The Thoracic Clinic, Sismanoglion Hospital, Athens, Greece.
In the last 10 years, we have operated on 33 cases of hydatid cysts of the liver with intrathoracic rupture and we have reviewed our cases.

\section{Review of cases}

Of the 33 patients, 23 were male and 10 female; 23 came from rural areas. The age incidence is shown in table 1.

Table 1 Age incidence of patients

\begin{tabular}{lc}
\hline Age & Number of cases \\
\hline Up to 40 years & 14 \\
$41-50$ & 8 \\
$51-60$ & 9 \\
Over 60 & 2 \\
\hline The sites of rupture are shown in table 2 and the \\
clinical features in table 3. \\
Table $2 \quad$ Sites of rupture \\
\hline Site & \\
\hline Rupture into the bronchi and biliary system & 21 \\
Rupture into the pleural cavity & 7 \\
Simultaneous rupture into the bronchi and & 4 \\
pleural cavity & 1 \\
\hline
\end{tabular}


Table 3 Clinical features

\begin{tabular}{lc}
\hline Feature & Number of cases \\
\hline Without symptoms, incidental finding & 1 \\
Irritating cough & 24 \\
Purulent sputum & 24 \\
Bile-stained sputum & 10 \\
Chest pain & 16 \\
Pyrexia & 20 \\
Shortness of breath & 11 \\
Vomique & 6 \\
Haemoptysis & 8 \\
Coughing up membranes & 6 \\
Allergic reaction & 2 \\
Finger clubbing & 2 \\
\hline
\end{tabular}

There is a variety of symptoms (table 3 ). Only one case had no symptoms. Irritating cough was the more common symptom (24 patients), with purulent sputum in 24 and bile-stained sputum in 10. Chest pain was a frequent symptom (10 patients) and pyrexia was found in 20 . This rise of temperature was probably caused by infected cysts. Shortness of breath was found in 11 patients. Sudden expectoration of pus and membranes of the cyst (vomique) occurred in six and coughing up daughter cysts of hydatid elements in six. Allergic reactions were noticed in only two. This is contrary to other statistics in which the percentage of allergic reaction is much higher. Finger clubbing was found in two cases of longstanding infected cysts.

The results of laboratory investigations are shown in table 4.

Table 4 Results of laboratory investigations

\begin{tabular}{lllr}
\hline Yest & $\begin{array}{l}\text { Number } \\
\text { of cases }\end{array}$ & Positive & Negative \\
\hline Intradermal test (Casoni) & 23 & 18 & 5 \\
Complement fixation test (Weinberg) & 23 & 16 & 7 \\
Eosinophilia (more than 6\%) & 33 & 10 & 23 \\
Pleural fluid (Hooks or Scolices) & 12 & 10 & 2 \\
Liver scan & 10 & 10 & - \\
\hline
\end{tabular}

\section{Table 5 Radiological results}

\begin{tabular}{|c|c|c|}
\hline \multicolumn{2}{|l|}{ Result } & Number of cases \\
\hline \multicolumn{3}{|c|}{ Round or oval shadow of varying size in the } \\
\hline \multicolumn{2}{|c|}{ RLL developing in the right dome of diaphragm } & 5 \\
\hline Apparent pleural cyst & & 5 \\
\hline $\begin{array}{l}\text { clearly separated from the diaphragm } \\
\text { blurred outline }\end{array}$ & $\begin{array}{l}3 \\
2\end{array}$ & \\
\hline High position of the right dome & & 6 \\
\hline Pneumonitis of RLL & & 14 \\
\hline Abscess formation & & 12 \\
\hline single & 8 & \\
\hline multiple & 4 & \\
\hline Pleural fluid & & 8 \\
\hline Spontaneous pyopneumothorax & & 3 \\
\hline Pulmonary dissemination & & 3 \\
\hline Bronchograms & & 8 \\
\hline
\end{tabular}
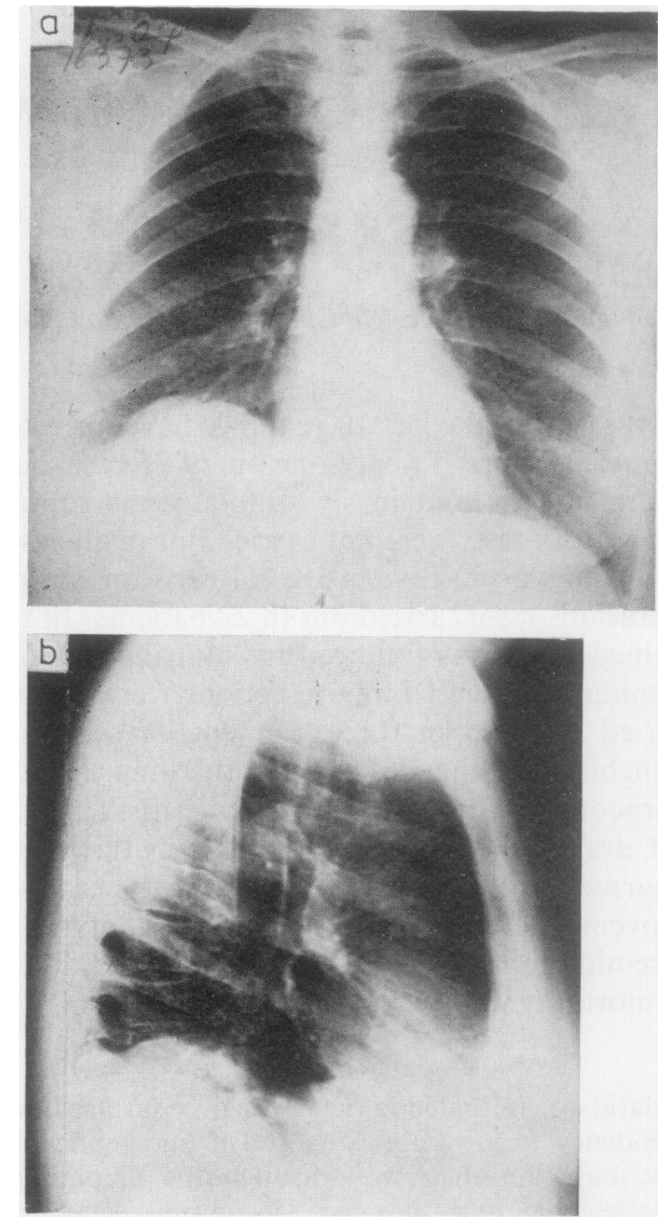

Fig 1 Hydatid cyst of the right lower lobe after rupture of a hepatic cyst into the bronchus.

Radiological findings are shown in table 5. A large hepatic cyst situated under the diaphragm is of particular interest to the thoracic surgeon (figs 1-2). It may be mistaken for a pulmonary cyst or may mimic a pleural effusion and be aspirated, with serious complications. Simple high dome of the diaphragm was noticed in six cases. An apparent pulmonary cyst either clearly separated from the diaphragm or with blurred outline was found in three and two respectively. Pneumonitis or abscess formation in the right lower lobe was noticed in 14 and 12 cases respectively. Pleural fluid was observed in eight. Spontaneous pyopneumothorax caused by simultaneous rupture of the cyst into the pleura and bronchi was noticed in three (figs 3-5). Pulmonary dissemination caused by daughter cyst formation was found in three. A bronchogram is a valuable examination for confirming irreversible bronchectatic changes. 


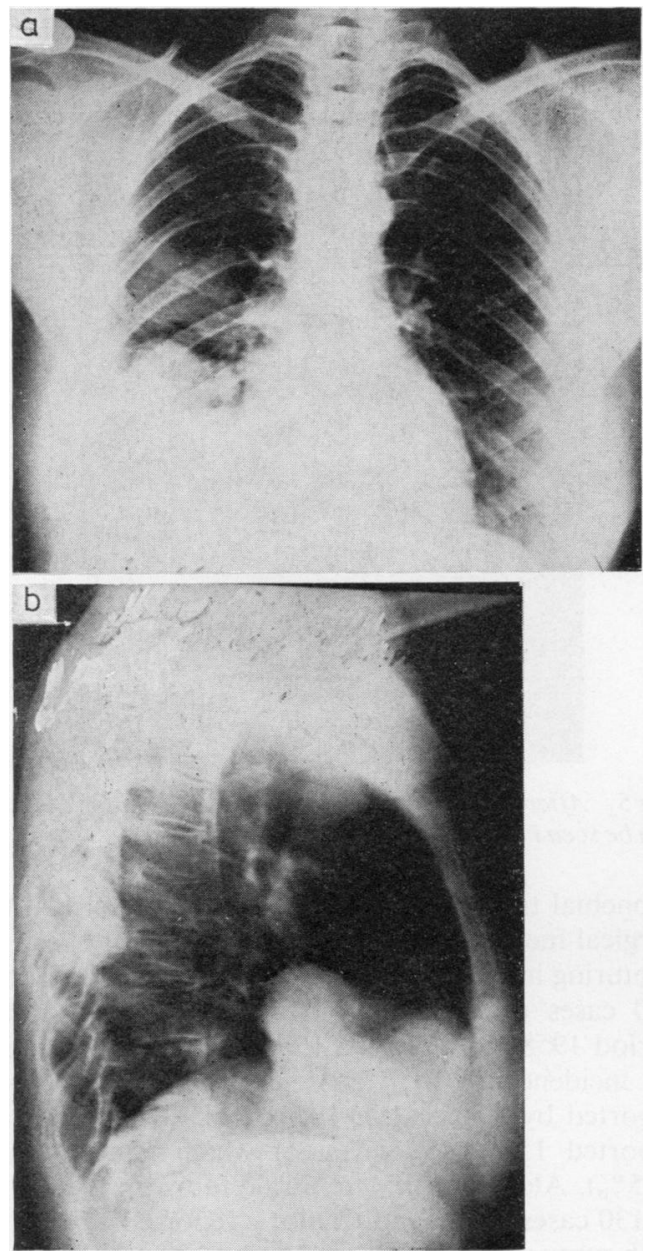

Fig 2 Thoracic involution of a hepatic cyst.

Table 6 Methods of surgery used

\begin{tabular}{lll}
\hline Method & Number of cases \\
\hline Conservative operation & 22 \\
Resections & 3 & 11 \\
Segmental & 8 & \\
Lobectomy & 0 & \\
Pneumonectomy & 0 & 5 \\
Decortication & & \\
\hline
\end{tabular}

The operative methods are shown in table 6 . In the conservative operation the hepatic cavity was evacuated and drained separately, followed by suturing of the diaphragmatic rupture and closure of the bronchial opening if present, with drainage of the pleural cavity. If there was a separate cyst of the lung this was removed together with a part of the adventitia, the fistula was closed, and the remaining pulmonary cavity sutured.
Lung resection was not done often because lung lesions are usually reversible, but in cases where the lung was seriously damaged resection was necessary. In our cases three segmental resections and eight lobectomies were needed. Pneumonectomy was not required.

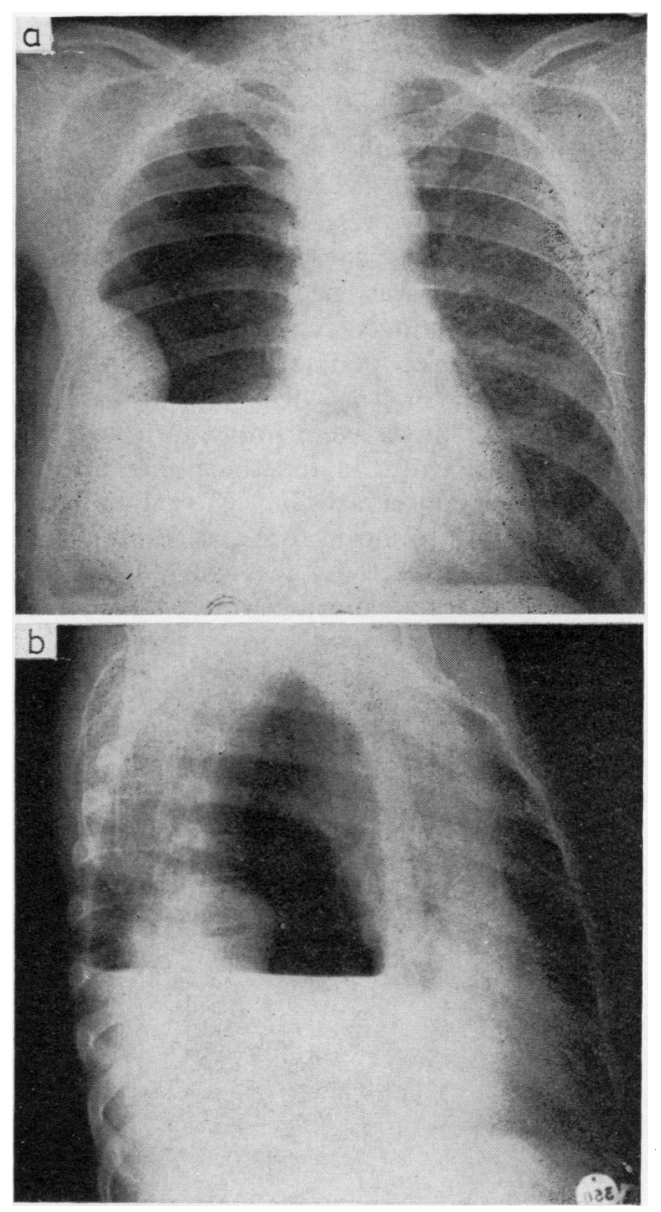

Fig 3 Rupture of a hepatic cyst into the pleura with hydropneumothorax. A parietal cyst can be seen.

Rupture of a cyst into the pleural cavity necessitates emergency thoracotomy. The objects of the operation then are to remove the parasite, drain the hepatic cavity, and re-expand the lung immediately. Separate drainage of the chest is necessary. Even in neglected rupture with pyothorax and daughter cysts in the visceral or parietal pleura the same type of operation is indicated. The danger of recurrence is greater than when cysts rupture into the bronchi but this danger does not justify pleuropneumonectomy. In cases with biliary complications opening 
Table 7 Outcome of operation

\begin{tabular}{lc}
\hline Outcome & Number of cases \\
\hline Immediate postoperative complications & \\
Haemorrhage & 2 \\
Empyema & 2 \\
Late postoperative complications & 5 \\
Recurrent haemoptysis & 1 \\
Persistent bile fistula & 3 \\
Dissemination of hydatic cyst & 20 \\
Without complications & 0 \\
Operative mortality & \\
\hline
\end{tabular}

of the common bile duct is necessary with T-tube drainage.

Early postoperative results are shown in table 7 . Postoperative haemorrhage occurred in two cases and thoracotomy was necessary in both. Postoperative empyema occurred in two cases and after simple drainage the course was uneventful. Recurrent haemoptysis was observed in five cases. Bronchiectatic changes were found in two of those and no obvious cause in the remaining three. All were treated conservatively. Dissemination of hydatid cysts was found in three, affecting the right lower lobe, and lobectomy was carried out in all. There was no operative mortality.

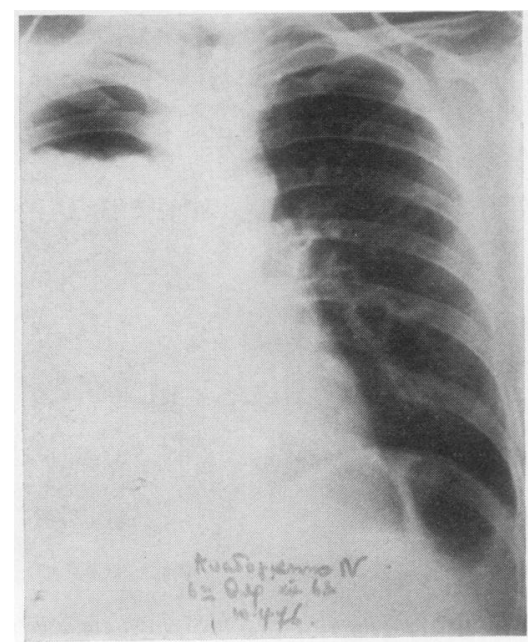

Fig 4 Rupture of hepatic cyst into the pleura. Daughter cysts floating on the surface.

\section{Discussion}

Cysts located in the dome of the liver can grow into the chest and cause severe complications. This extension of a cyst into the thorax is usually caused by its position in the superior aspect of the liver and the attendant inflammatory reaction. It is seen in approximately $3 \%$ of the cases. ${ }^{3}$ Stathatos et al reported 18 cases, in 15 of which rupture into the

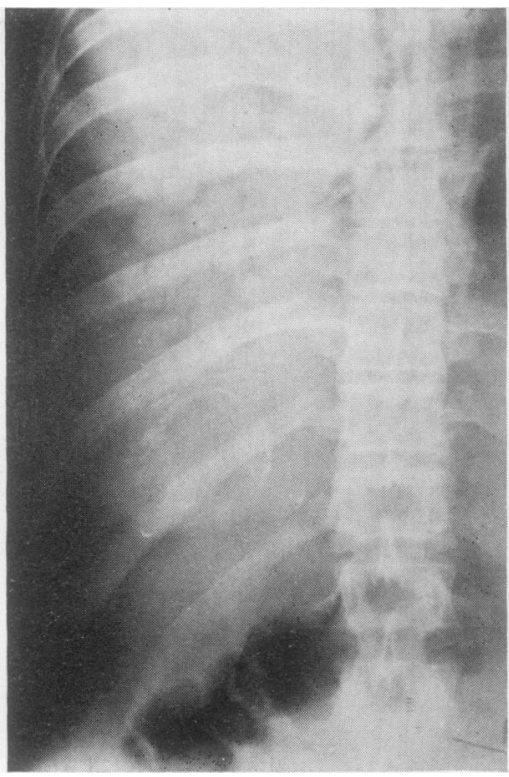

Fig 5 After removing the fluid a mass of daughter cysts can be seen in the pleural and hepatic cavity.

bronchial tree occurred. ${ }^{7}$ Tsoufis et al described the surgical methods and results in 28 cases of liver cyst rupturing into the bronchus. ${ }^{6}$ Guedj et $a l^{13}$ reporting 600 cases of hydatid cysts of the liver from the period 1958-68, observed 15 cases of rupture giving an incidence of $2.5 \%$. A similar percentage was reported by Kourias ${ }^{14}$ in 1334 cases. Bourgeon et al ${ }^{15}$ reported 154 cases, seven of which had ruptured $(4 \cdot 5 \%)$. Aletras et al $^{12}$ reported four ruptures $(3 \%)$ in 130 cases. Deve and Caniot ${ }^{16}$ reported 1743 cases with rupture in $4.5 \%$.

When the hydatid cyst communicates with the biliary system and becomes infected, the inflammatory process extends towards the inferior aspect of the diaphragm and obliterates the space between liver and diaphragm. As the size of the cyst increases the diaphragmatic fibres get thinner from the pressure and eventually rupture. If there are preexisting adhesions in the pleural cavity then the rupture is directed into the bronchial tree. In cases where there are no pleural adhesions the rupture may appear only in the pleural cavity, but this is less common. ${ }^{3}$ A true reactive bronchopneumonitis occurs when the cyst opens into a bronchus and a broncho-biliary fistula is created. This results in pain and expectoration of bile-stained pus sometimes with daughter cysts or membranes.

The diagnosis of rupture presents no difficulty when bile-stained sputum and daughter cysts are expectorated. Daughter cysts are found more frequently in hepatic than in pulmonary cysts. 
The infected purulent cysts are usually old chronic lesions; communication with the bile ducts is blocked by elements of hydatid cyst and jaundice may appear.

Primary rupture into the pleural cavity without lung involvement is rare. Of our 33 cases only six ruptured into the pleura.

By the time the final rupture occurs the diaphragm has usually become attached to both the liver and the lung. The symptoms are of a sudden tearing pain, dyspnoea, and anaphylactic shock from which the patient may die. If he recovers, the chest radiograph shows a pleural effusion or a pyopneumothorax particularly if the rupture into the pleura occurred after rupture into the bronchus.

Many authors are in favour of a transpleural approach. ${ }^{138-10}$ We have followed the same approach. It gives a good exposure and the lung complications can be treated. If abdominal complications occur the incision can be extended to the abdomen or a separate abdominal incision made. ${ }^{50}$

Philippakis reports six cases, in four of which rupture occurred into the middle lobe. ${ }^{17} \mathrm{~A}$ thoracoabdominal incision was used in four and in two a combination of thoracic and abdominal incision was used. Avlamis ${ }^{11}$ stresses the importance of draining the common bile duct in ruptured cyst with biliary complications. We prefer to be as conservative as possible as in our cases of hydatid disease of the chest. ${ }^{18}$

After dissecting the bronchial fistula the opening in the diaphragm is enlarged and the hepatic cavity cleared and drained subdiaphragmatically. The middle and lower lobe can undergo an intense suppurating process that makes the surgeon resort to resection, which can be technically difficult.

It is important to be conservative in dealing with pulmonary complications and we agree with Crausaz ${ }^{3}$ that lung complications are, in the majority, reversible-Crausaz, in 25 cases, carried out only one resection. ${ }^{3}$ The drainage of the hepatic cavity must be continued until complete closure of the cavity occurs. The diaphragm is closed in layers and a water seal drainage catheter is left in the pleural cavity for 48 hours at least.

When the common bile duct is obstructed by elements of hydatid cyst a T-tube has to be inserted at the same operation.

A cyst rupturing into the pleural cavity requires emergency thoracotomy after the anaphylactic reaction is over. The objects of the operation are to remove the cyst which is usually found floating in the pleural cavity. If there are remnants of the cyst in the hepatic cavity it has to be drained as already described.

Even in neglected rupture with pyopneumothorax and daughter cysts in the parietal or visceral pleura the same type of operation is indicated with pleurectomy and decortication of the lung. The danger of recurrence is greater than in ruptured cysts into the bronchial tree but even this danger does not justify pneumonectomy.

\section{References}

1 Susman MP. Hydatid disease as it affects the thoracic surgeon. J Thorac Surg 1953;26:111-30.

2 Tool H, Propatoridis J, Pangalos N. Intrapulmonary rupture of hydatid cyst of the liver. Thorax 1953;8 274-80.

3 Crausaz PH. Hydatid cyst of the lung and hydatid disease of the liver with intra-thoracic evolution. $J$ Thorac Cardiovasc Surg 1967;53:116-29.

4 Kourias B, Mantonakis S. Kystes hydatique du foie rompus dans les voies biliaires. Regles thèrapeutiques actuelles. J Chir (Paris) 1968;96:21-36.

5 Maragos G. The surgical treatment of complicated hydatid cyst of the liver. Sixth Panhellenic Congress of Surgery 1968;VI:270-86.

6 Tsoufis E, Agorogiannis S, Kourmadas C, Papaioannou K. Surgical methods and results in 28 cases of hydatid cyst of the liver ruptured to the bronchi. Sixth Panhellenic Congress of Surgery 1968;VI:377-85.

7 Stathatos C, Kontaxis A, Zaphiracopoulos P. Hydatid cyst of the liver. Sixth Panhellenic Congress of Surgery 1968;VI:365-70.

8 Zaphiracopoulos P, Pappas C, Rouskas G. The management of thoracic complications of hepatic cyst ruptured into the chest. Sixth Panhellenic Congress of Surgery $1968 ; \mathrm{VI}: 537-46$.

9 Giannacopoulos G, Papamichel E. Hydatid disease of the liver. Chest complications. Sixth Panhellenic Congress of Surgery 1968;VI:508-12.

10 Katsaras E, Zoitopoulos M, Katsara R. Observations of trans-thoracic surgical approach of hydatid cyst of the upper surface of the liver. Sixth Panhellenic Congress of Surgery 1968;VI:435-40.

11 Avlamis G, Bonelos K. Problems and surgical treatment of broncho-biliary fistulas due to hydatid cyst of the liver. Second Panhellenic Congress of Surgery 1961;II:960-4.

12 Aletras H, Dadoukis J, Aidonopoulos A. Bronchobiliary fistulas. Galenus 1971 ; $13: 657-63$.

13 Guedj P, Gairoard G, Morvan F, Bogaert J. Le traitement chirurgical actuel du kyste hydatique du foie et ses principales complications.J Chir (Paris) 1967; 93:191-7.

14 Kourias V, Chouliaras C, Gikas D. Thoughts and conclusions in 1334 operated cases of hydatid of the liver Sixth Panhellenic Congress of Surgery 1968;VI:371-6.

15 Bourgeon et al. Reported by Tsoufis E et al. Sixth Panhellenic Congress of Surgery 1968;VI:377-85.

16 Deve F. L'ouverture de kysts hydatique du foie dans les poumons et les bronches. Cinquieme Congres Annuel des Sciences Medicales 1935. Oran: 27-32.

17 Philippakis M, Chourdakis C, Alivizatos C, Iconomidis C. Hepato-bronchial fistula due to ruptured hydatid cyst of the upper part of the liver. Sixth Panhellenic Congress of Surgery 1968; VI:441-5.

18 Xanthakis D, Efthimiadis M, Papadakis G et al. Hydatid disease of the chest. Thorax 1972;27:517-28. 